\title{
Prevalence and Impact of Metabolic Syndrome on Short-Term Prognosis in Patients with Acute Coronary Syndrome: Prospective Cohort Study
}

\author{
Korinan Fanta $\mathbb{D}^{\prime}$ \\ Fekede Bekele Daba (ID) \\ Elsah Tegene Asefa ${ }^{2}$ \\ Legese Chelkeba iD ${ }^{3}$ \\ Tsegaye Melaku (D) \\ 'Department of Clinical Pharmacy, \\ Institute of Health, Jimma University, \\ Jimma, Oromia, Ethiopia; ${ }^{2}$ Department of \\ Internal Medicine, Institute of Health, \\ Jimma University, Jimma, Oromia, \\ Ethiopia; ${ }^{3}$ Department of Pharmacology \\ and Clinical Pharmacy, College of Health \\ Science, Addis Ababa University, Addis \\ Ababa, Ethiopia
}

Correspondence: Korinan Fanta Department of Clinical Pharmacy, Institute of Health, Jimma University, P.O.Box: 378, Jimma, Ethiopia

Tel +251911598485

Fax +25I 476617980

Email korif53@gmail.com
Purpose: Despite the increasing burden of metabolic syndrome (MS) and ischemic heart disease in sub-Saharan Africa, data on the prevalence of MS among patients with acute coronary syndrome (ACS) from the regions are limited. Hence, this study is aimed to evaluate the prevalence and impact of MS on 30-day all-cause mortality in patients hospitalized with ACS.

Patients and Methods: We prospectively assessed 176 ACS patients, who were admitted to two tertiary hospitals in Ethiopia. MS was diagnosed based on a harmonized definition of MS. In-hospital major adverse cardiovascular events (MACE) and 30-day mortality were recorded. Multivariable cox-regression was used to identify predictors of 30-day mortality. Results: Among 176 ACS patients enrolled, 62 (35.2\%) had MS. Majority of the patients $(62.5 \%)$ were male with the mean age of $56 \pm 11.9$ years. ACS patients with MS were older, presented with atypical symptoms, and they had history of hypertension, diabetes, dyslipidemia and coronary artery disease compared to those without MS. MS was also significantly associated with in-hospital MACE (30.6\% vs 17.5\%; $\mathrm{p}=0.046)$ and 30-day mortality [adjusted hazard ratio $(\mathrm{AHR})=3.25,95 \% \mathrm{CI}=1.72-6.15$ ]. The other significant predictors of 30-day mortality were pre-hospital delay $>12 \mathrm{~h}(\mathrm{HR}=4.32,95 \% \mathrm{CI}=1.68-11.100)$, killip class $\geq 2 \quad(\mathrm{HR}=10.7,95 \% \mathrm{CI}=2.54-44.95)$, and ejection fraction $<40 \quad(\mathrm{HR}=2.5995 \%$ $\mathrm{CI}=1.39-4.84)$.

Conclusion: The prevalence of MS among patients with ACS in Ethiopia is high. MS was significantly associated with high in-hospital MACE and it was an independent predictor of 30-day mortality. Initiating appropriate strategies on MS prevention and timely diagnosis of MS components could decrease the burden of ACS and improve patient's outcome.

Keywords: metabolic syndrome, myocardial infarction, mortality, sub-Saharan Africa

\section{Introduction}

The metabolic syndrome (MS) represents a group of correlated metabolic disorders that have a synergic effect on atherosclerotic cardiovascular disease risk. ${ }^{1,2}$ According to the National Cholesterol Education Program Adult Treatment Panel III (NCEP/ATP III), MS is defined by the presence of any three of the following metabolic abnormalities: abdominal obesity, dyslipidemia, hypertension or treated hypertension, and elevated fasting plasma glucose levels. ${ }^{3}$

About one-quarter of the world population is currently affected by MS. ${ }^{4}$ Contrary to the prior thought, MS are no longer rare in sub-Saharan Africa (SSA). Recent meta-analysis of a community-based study reported that the overall 
prevalence of MS in SSA was $17.1 \%$ and $18 \%$ according to the NCEP/ATP III and international diabetes federation (IDF) diagnostic criteria, respectively. ${ }^{5}$ Furthermore, the burden of non-communicable disease in SSA regions is exceeding the global average. ${ }^{6}$ Historically, rheumatic heart disease from infections source has been the leading cause of cardiovascular disease, while other causes were comparatively rare. ${ }^{6,7}$ However, recent studies show that stroke and ischemic heart disease are the leading cause of cardiovascular disease burden in the regions. ${ }^{5,6,8}$ These findings reflect that MS, a common precursor of these cardiovascular diseases is common in the continent. However, data on the prevalence of MS among ischemic heart disease particularly, ACS patients in the region are limited and the available data on MS are mainly done in diabetic, HIV/AIDS and hypertensive patients. ${ }^{9}$

Many studies have shown that MS has a negative impact on the prognosis of patients who survived ACS. ${ }^{10-12}$ These studies were predominantly conducted in developed countries among ACS patients managed in different ways which undoubtedly influenced prognosis and the occurrence of new adverse outcomes among these patients. However, an association of MS with risk of cardiovascular disease and mortality outcomes varies according to race/ethnicity, geographical area, age, and access to medical care. ${ }^{13}$ Despite these pieces of evidences, data on the association between MS and the outcome of ACS patients from SSA is rare. Therefore, we evaluated the prevalence and impact of MS 30-day allcause mortality among patients hospitalized with ACS managed medically in tertiary hospitals in Ethiopia.

\section{Methods}

\section{Study Design and Study Population}

A prospective cohort study was conducted at two tertiary hospitals in Ethiopia (Saint Peter's Specialized Hospital and Jimma University Medical Center) from March 2018 to November 2018. In the present study, ACS was diagnosed according to the third universal definition of myocardial infarction. ${ }^{14}$ Consecutive patients with confirmed ACS diagnosis admitted to selected hospitals who fulfilled the following eligibility criteria were enrolled: (1) age $\geq 18$ years; (2) willing to provide written or oral informed consent; (3) type I myocardial infarction. Patients who died before clinical assessment or anthropometric and biochemical measurements were excluded. Additionally, readmitted patients (if enrolled previously), and patients with initial ACS diagnosis changed were excluded from the present study (see Supplementary Figure 1). The study protocol was approved by Institutional Review Board (IRB) of Jimma University, the Institute of Health before initiating data collection with a reference number of IHRPGD/193/18.

\section{Metabolic Syndrome Diagnosis and Scores}

Metabolic syndrome was assessed based on the harmonized definition of MS outlined by the IDF Task Force on Epidemiology and Prevention, NHLBI, AHA, World Heart Federation, International Atherosclerosis Society, and International Association for the Study of Obesity ${ }^{15}$ and ethnic-based waist circumference as defined by $\operatorname{IDF}^{16}$ was used. According to this criterion patient with ACS were diagnosed to have MS if they have at least three of the following components: (1) Elevated waist circumference ( $\geq 94 \mathrm{~cm}$ for men or $\geq 80 \mathrm{~cm}$ for women); (2) elevated fasting plasma glucose (FPG) $\geq 100 \mathrm{mg} / \mathrm{dl}$ or on drug treatment for raised glucose; (3) elevated blood pressure $\geq 130 / 85$ $\mathrm{mmHg}$ or on antihypertensive treatment for a patient with a history of hypertension (4) reduced high-density lipoprotein cholesterol (HDL-C) $[<40 \mathrm{mg} / \mathrm{dL}(1.03 \mathrm{mmol} / \mathrm{L})$ in males and $<50 \mathrm{mg} / \mathrm{dL}(1.29 \mathrm{mmol} / \mathrm{L})$ in females] or and (5) Raised triglycerides $\geq 150 \mathrm{mg} / \mathrm{dL}(1.7 \mathrm{mmol} / \mathrm{L})$. We did not consider treatment with lipid-lowering drugs as a criterion for patients on lipid-lowering agents for the sole purpose of cardiovascular disease prevention.

Waist circumference was measured at the midway between the lower margin of the ribs and the upper margin of the iliac crest in a horizontal plane using plastic metric tape. ${ }^{16}$ Brachial blood pressure was measured twice by appropriate sized-cuffs in a sitting position and a mean of the two recordings was used in the analyses. Fasting venous blood sample was collected for biochemical measurement in the morning after 8-12 fasting overnight. Accordingly, fasting plasma glucose, high-density lipoprotein, triglyceride low-density lipoprotein, and total cholesterol were measured using Cobas ${ }^{\circledR} 6000$ analyzer.

\section{Data Collection}

Data collection was undertaken by trained health professionals who interviewed the study participants using pretested structured questionnaires and examined using standardized instruments and methods. Data collectors also abstracted relevant information (presenting 
symptoms, clinical data at admissions, cardiovascular risk factors and treatment) from active patient's medical records prospectively.

The primary end-points of the study were 30-day allcause mortality and in-hospital MACE. The data on primary end-points were collected by checking medical documentation on a daily basis from admission to discharge or death prospectively. For patients who died during hospitalization, physician's death summary notes were reviewed. For those who died after hospital discharge, telephone contact with family members or caregivers and a death certificate review was used to confirm death. The definitions of clinical endpoints used in the present study are available in (Supplementary Material)

\section{Operational Definitions}

Major adverse cardiovascular events (MACE): defined as a composite of in-hospital cardiovascular death, stroke, and non-fatal acute myocardial infarction (re-infarction).

Typical chest pain: defined as a retrosternal sensation of pain/discomfort ("angina") radiating to either or both arm, neck, or jaw, which may be intermittent (usually lasting $\geq 10$ minutes) or persistent. It might be accompanied by additional symptoms such as nausea, abdominal pain, sweating, and syncope.

Atypical presentation: was defined as a non-classic symptoms, including epigastric pain, indigestion-like symptoms, stabbing or pleuritic pain, and isolated dyspnea in the absence of typical chest pain.

\section{Statistical Analysis}

Data were entered into Epidata version 4.2 and analyzed using statistical package for social science, version 23 (IBM, Armonk, NY, USA). Categorical variables were presented as proportion and compared between ACS with and without MS using the chi-square test. Continuous variables were presented as mean \pm standard deviation when normally distributed and median (interquartile range) when skewed. Continuous variables were compared using the Student's $t$-test or Mann-Whitney test as appropriate. The 30-day mortality rate among ACS patients with and without MS was compared by using Kaplan-Meier survival curve and Log rank test. Cox regression was used to identify independent predictors of time to 30-day mortality. P-value $<0.25$ was used as a cutoff point to select candidate variables on binary cox-regression. Multivariable cox-regression with backward step-wise methods was used to identify the independent predictors of 30-day mortality of ACS patients.
Two-tailed p-values $<0.05$ were considered as a statistically significant difference.

\section{Results}

\section{Baseline Clinical Characteristics and Metabolic Syndrome Components}

Among 176 ACS patients studied, 62 (35.2\%) had MS. Patients with MS were older $(58.5 \pm 11.0$ vs $54.6 \pm 12.2, \mathrm{p}=$ 0.035) compared to those without MS. Similarly, ACS patients with MS were more likely to have a prior history of hypertension, diabetes, dyslipidemia, chronic kidney disease, and coronary artery disease $(\mathrm{p}=0.001)$. Overall, there was no a statistically significant difference between ACS patients with MS and those without MS regarding sex, residence, and behavioral measures such as smoking and alcohol use (Table 1). The most common components of MS identified were abdominal obesity ( $46 \%$ ) and a history of hypertension or blood pressure $>130 / 85 \mathrm{mmHg}$ (45.5\%) (Figure 1).

\section{Clinical Presentation, Biochemical Data and Key Diagnostics}

Patients with MS were more likely to present with atypical symptoms $(\mathrm{p}=0.026)$ and high admission systolic blood pressure $(p=0.007)$ compared to those without MS. Likewise, patients with MS were also present with high admission fasting plasma glucose, triglyceride, low-density lipoprotein cholesterol, and total cholesterol compared to those without MS (p-value $<0.001$ ). There was no significant difference between ACS patients with MS and without MS in ACS subtypes and other clinical findings (Table 2).

\section{Treatment and In-Hospital Outcomes}

Overall, aspirin, dual antiplatelet (aspirin and clopidogrel), and statins were used in more than $90 \%$ of ACS patients. Patients with MS were more likely to receive dual antiplatelet therapy compared to those without MS ( $\mathrm{p}=0.015)$. The use of beta-blockers, ACEI/ARB, and any heparin was sub-optimal (70-80\%), and no significant difference between the two groups (Table 3). Patients with MS were more likely to develop MACE during their hospital stay $(\mathrm{p}=0.026)$. In particular, patients with MS were more likely to develop heart failure $(\mathrm{p}=0.017)$ and cardiogenic shock ( $\mathrm{p}=0.015$ ) compared to those without MS. However, there was no difference regarding cardiovascular death, non-fatal stroke and re-infarction between the two groups (Table 3). Discharge medications follow similar patterns to 
Table I Demographics and Baseline Characteristics in Patients with and without MS Presented with ACS

\begin{tabular}{|c|c|c|c|c|}
\hline Baseline Characteristics & MS $(n=62)$ & No MS $(n=1 \mid 4)$ & All Patients $(n=176)$ & P-value \\
\hline Age (years), mean $\pm S D$ & $58.5 \pm 11.0$ & $54.6 \pm 12.2$ & $56.0 \pm 11.9$ & 0.035 \\
\hline Sex (Male), n (\%) & $39(62.9)$ & $71(62.3)$ & $110(62.5)$ & 0.935 \\
\hline \multicolumn{5}{|l|}{ Residence, n (\%) } \\
\hline Urban & $49(79.0)$ & $86(75.40)$ & $135(76.7)$ & 0.590 \\
\hline Rural & $13(2 \mid .0)$ & $28(24.6)$ & $4 \mathrm{I}(23.3)$ & \\
\hline \multicolumn{5}{|l|}{ Educational status, n (\%) } \\
\hline Unable to $\mathrm{read} / \mathrm{write}$ & $18(29.0)$ & $38(33.3)$ & $56(31.8)$ & 0.848 \\
\hline Read and write & $16(25.8)$ & $24(2 I . I)$ & $40(22.7)$ & \\
\hline Primary education & II (I7.7) & $18(15.8)$ & $29(16.5)$ & \\
\hline Secondary and above & $17(27.4)$ & $34(29.8)$ & $51(29.0)$ & \\
\hline \multicolumn{5}{|l|}{ Occupational status, n (\%) } \\
\hline Employee & $17(27.4)$ & $26(22.8)$ & $43(24.4)$ & 0.477 \\
\hline Farmer/labor workers & $24(38.7)$ & $55(48.2)$ & $79(44.9)$ & \\
\hline Unemployed/retired & $21(33.9)$ & $33(28.9)$ & $54(30.7)$ & \\
\hline Smoking, n (\%) & $9(14.5)$ & $19(16.7)$ & $28(15.9)$ & 0.709 \\
\hline Alcohol use, n (\%) & $19(30.6)$ & $34(29.8)$ & $53(30.1)$ & 0.910 \\
\hline Hypertension, n (\%) & $4 I(66.1)$ & $33(28.9)$ & $74(42.0)$ & $<0.001$ \\
\hline Diabetes, n (\%) & $35(56.5)$ & II (9.6) & $46(26.1)$ & $<0.001$ \\
\hline Dyslipidemia*, n (\%) & $43(69.4)$ & $47(4 I .2)$ & $90(51.1)$ & $<0.001$ \\
\hline History of CAD, n (\%) & $22(35.5)$ & $15(13.2)$ & $37(21.0)$ & 0.001 \\
\hline CKD, n (\%) & $12(19.4)$ & $5(4.4)$ & $17(9.7)$ & 0.001 \\
\hline
\end{tabular}

Abbreviations: CAD, coronary artery disease; CKD, chronic kidney disease; MS, metabolic syndrome; SD-standard deviation.

in-hospital medications and comparable between both groups (Table 3).

\section{Predictors of 30-Day Mortality}

From the total the total of 176 ACS patients, 46 (26.1\%) patients were died during the 30-day follow-up. Twelve variables (age, residence, pre-hospital delay, presentation symptoms, systolic blood pressure, heart rate, serum creatinine, hemoglobin, Killip class, ACS subtypes, MS, and ejection fraction) with $\mathrm{p}$-value $<0.25$ on bivariate cox-regression were included in the final model of multivariable cox-regression. On multivariable Cox proportional hazard model analysis, pre-hospital delay $>12 \mathrm{~h}$, systolic blood pressure, Killip class $\geq 2$, presence of MS, and low ejection fraction were significantly associated with 30 -day mortality (Table 4 ).

In this study patients who were presented to hospital after $12 \mathrm{~h}$ of symptom onset were about four times more likely to have 30-day mortality compared to those presented within $12 \mathrm{~h}$ of symptom onset [hazard ratio (HR) $=4.3295 \%$ confidence interval $(\mathrm{CI})=1.68-11.10]$. Patients presented with killip class $\geq 2$ had about 11-fold increased hazard of 30 day mortality compared to those patients presented with Killip class $1(\mathrm{HR}=10.7,95 \% \mathrm{CI}=2.54-44.95)$. Similarly, ACS patients with MS were about 3 times more likely to have 30-day mortality than ACS patients without MS (HR $=3.25,95 \% \mathrm{CI}=1.72-6.15)$. Likewise, patient who had low ejection fraction $(<40 \%)$ had three-fold increase in 30-day mortality compared to those who had preserved ejection fraction $(\mathrm{HR}=2.5995 \% \mathrm{CI}=1.39-4.84)$. High admission systolic blood pressure was associated with good prognosis (HR $=0.98,95 \%$ CI $=0.96-0.99)$ (Table 4). Kaplan Meier cumulative 30-day survival curve was compared between ACS patients with MS and without MS using Log rank test $(\mathrm{p}=0.004)$ (Figure 2).

\section{Discussion}

This study, the first in Ethiopia, showed a high prevalence of MS among patients with ACS. Overall, individuals with 


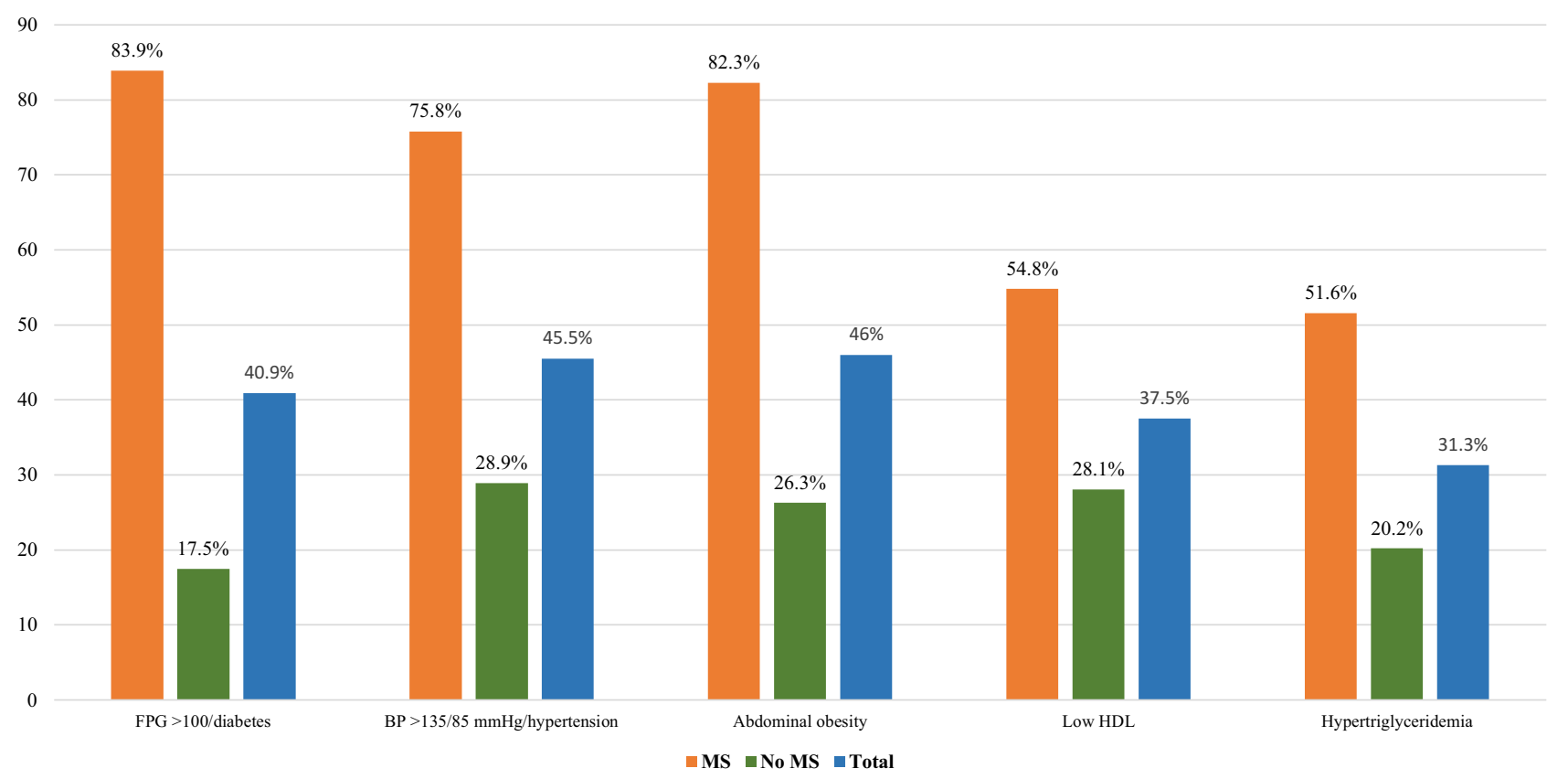

Figure I

MS were more likely to be older, present with atypical symptoms, and have a history of hypertension, diabetes dyslipidemia, chronic kidney disease, and coronary artery disease before the index event. In the present study, MS is associated with in-hospital MACE and 30-day all-cause mortality. Even after adjusting for confounders, MS was still an independent predictor of 30-day all-cause mortality.

Table 2 Clinical Presentation, Laboratory Measures, and Key Diagnostics in Patients with ACS, Stratified According to Presence or Absence of MS

\begin{tabular}{|c|c|c|c|c|}
\hline Parameters & MS $(n=62)$ & No MS $(n=1 \mid 4)$ & All Patients $(n=176)$ & P-value \\
\hline Pre-hospital delay $>12 \mathrm{~h} \mathrm{n}(\%)$ & $42(67.7)$ & $77(67.5)$ & $119(67.6)$ & 0.979 \\
\hline Typical chest pain, n (\%) & $32(51.6)$ & $76(66.7 \%)$ & $108(61.4)$ & 0.026 \\
\hline Atypical presentation, $\mathrm{n}(\%)$ & $30(50.0)$ & $38(333)$ & $68(38.6)$ & \\
\hline Killip class $\geq$ II, n (\%) & $40(64.5)$ & $66(58.0)$ & $106(60.2)$ & 0.391 \\
\hline STEMI, n (\%) & $39(63.0)$ & $68(59.6)$ & $107(60.8)$ & 0.673 \\
\hline NSTE-ACS, n (\%) & $23(37.1)$ & $46(40.4)$ & 69 (39.2) & \\
\hline Positive cardiac biomarkers, n (\%) & $58(93.5)$ & $98(86.0)$ & $156(88.6)$ & 0.130 \\
\hline Systolic BP $(\mathrm{mmHg})^{\dagger}$ & $132 \pm 23.6$ & $121 \pm 24.9$ & $125 \pm 24.4$ & 0.007 \\
\hline Heart rate $(\mathrm{bpm})^{\dagger}$ & $91.6 \pm 24.8$ & $92.7 \pm 24.2$ & $92.3 \pm 24.3$ & 0.791 \\
\hline Serum creatinine $(\mathrm{mg} / \mathrm{dL})^{\dagger}$ & $1.18 \pm 0.84$ & $1.05 \pm 0.75$ & $1.10 \pm 0.78$ & 0.309 \\
\hline Hemoglobin $(\mathrm{mg} / \mathrm{dL})$ & $13.2 \pm 2.3$ & $14.0 \pm 2.7$ & $13.7 \pm 2.6$ & 0.05 \\
\hline $\mathrm{HDL} C(\mathrm{mg} / \mathrm{dL})^{\dagger}$ & $42.6 \pm 9.3$ & $45.4 \pm 9.6$ & $44.5 \pm 9.6$ & 0.066 \\
\hline LDL-C $(\mathrm{n}=170)(\mathrm{mg} / \mathrm{dL})^{\dagger}$ & $124.7 \pm 42.3$ & $105.4 \pm 34.8$ & $112.3 \pm 38.6$ & 0.002 \\
\hline Cholesterol $(n=169)(\mathrm{mg} / \mathrm{dL})^{\dagger}$ & $206.6 \pm 70.4$ & $169.1 \pm 48.2$ & $182 \pm 59.5$ & $<0.001$ \\
\hline Triglyceride $(\mathrm{mg} / \mathrm{dL})^{\ddagger}$ & $152(10 \mid-204.5)$ & $120(89-148)$ & $125.3(90.6-163.0)$ & 0.001 \\
\hline Fasting glucose $(\mathrm{mg} / \mathrm{dL})^{\ddagger}$ & $146.5(\mid 14.7-178.5)$ & $95.5(87.0-98.2)$ & $98(92-140)$ & $<0.001$ \\
\hline Ejection fraction $<40 \%(n=170)$ & $22(35.5)$ & $46(40.4)$ & $68(38.6)$ & 0.527 \\
\hline Diagnostic angiogram, n (\%) & $21(35.0)$ & $55(47.4)$ & $76(43.2)$ & 0.115 \\
\hline
\end{tabular}

Notes: ${ }^{\dagger}$ expressed as median and standard deviations (SD); ${ }^{\dagger}$ expressed as median and interquartile range (IQR).

Abbreviations: ACS, acute coronary syndrome; BP, blood pressure; bpm, beat per minute; HDL-C, high density lipoprotein-cholesterol; LDL-C, low density lipoproteincholesterol; MS, metabolic syndrome; NSTE-ACS-, non-ST-elevation acute coronary syndrome; STEMI, ST-elevation myocardial infarction. 
Table 3 Managements and In-Hospital Complications in Patients Presented with ACS with and without MS

\begin{tabular}{|c|c|c|c|c|}
\hline Variables & MS $(n=62)$ & No MS $(n=|| 4)$ & All Patients $(n=\mid 76)$ & P-value \\
\hline \multicolumn{5}{|l|}{ In-hospital medications, n (\%) } \\
\hline Aspirin & $62(100)$ & $113(99.1)$ & $175(99.4)$ & 0.460 \\
\hline DAPT & $61(98.4)$ & $100(87.7)$ & $161(91.5)$ & 0.015 \\
\hline Statin & $62(100)$ & III (97.4) & $173(98.3)$ & 0.198 \\
\hline Beta-blocker & $52(84.0)$ & $89(78.1)$ & $|4|(80.1)$ & 0.375 \\
\hline ACEI/ARB & $46(74.2)$ & $80(70.2)$ & $126(7 \mid .6)$ & 0.572 \\
\hline Any heparin & $5 I(82.3)$ & $83(73.0)$ & $134(76.1)$ & 0.160 \\
\hline Nitrates & $19(30.6)$ & $33(28.9)$ & $52(29.5)$ & 0.814 \\
\hline Morphine & $31(50.0)$ & $42(36.8)$ & $63(41.5)$ & 0.091 \\
\hline Diuretics & $35(56.5)$ & $59(51.8)$ & $94(53.4)$ & 0.551 \\
\hline $\mathrm{PCl}$ & $3(5.0)$ & $10(8.6)$ & $13(7.4)$ & 0.547 \\
\hline \multicolumn{5}{|l|}{ In -hospital complications } \\
\hline Cardiovascular death, n (\%) & $14(22.6)$ & $15(13.2)$ & $29(16.5)$ & 0.107 \\
\hline Stroke, n (\%) & $2(3.2)$ & $3(2.6)$ & $5(2.8)$ & 0.821 \\
\hline Re-infraction, n (\%) & $3(4.8)$ & $7(6.1)$ & $10(5.7)$ & 0.722 \\
\hline Acute heart failure, $\mathrm{n}(\%)$ & $10(16.1)$ & $6(5.3)$ & $16(9.1)$ & 0.017 \\
\hline Cardiogenic shock, n (\%) & II (I7.7) & $7(6.1)$ & $18(10.2)$ & 0.015 \\
\hline Major bleeding, n (\%) & $3(4.8)$ & $4(3.5)$ & $7(4.0)$ & 0.706 \\
\hline MACE, $\mathrm{n}(\%)$ & $19(30.6)$ & $20(17.5)$ & $39(22.2)$ & 0.046 \\
\hline Discharge Medications, n (\%) & $M S(n=44)$ & No-MS $(n=95)$ & All patients $(n=139)$ & \\
\hline Aspirin & $43(97.7)$ & $93(97.7)$ & $136(97.8)$ & 0.950 \\
\hline Clopidogrel & $35(79.5)$ & $72(75.8)$ & $107(77.0)$ & 0.625 \\
\hline Statin & $42(95.5)$ & $92(96.8)$ & $134(96.4)$ & 0.683 \\
\hline Beta-blocker & $36(82.0)$ & $82(86.3)$ & $118(84.9)$ & 0.491 \\
\hline ACEI/ARB & $36(82.0)$ & $69(72.6)$ & $105(75.5)$ & $0.24 I$ \\
\hline Spironolactone & $7(15.9)$ & $21(22.1)$ & $28(20.1)$ & 0.397 \\
\hline
\end{tabular}

Notes: aMACE (composite of cardiovascular death, non-fatal stroke, and re-infraction).

Abbreviations: ACEI, Angiotensin converting enzyme inhibitors ARB, Angiotensin receptor blocker; DAPT, Dual antiplatelet therapy (aspirin + clopidogrel); MACE, Major adverse cardiovascular events; MS, metabolic syndrome; PCl, Percutaneous coronary intervention.

Table 4 Predictors of 30-Day Mortality in Patients Presented with ACS

\begin{tabular}{|c|c|c|c|c|c|c|}
\hline \multirow[t]{2}{*}{ Variables } & \multicolumn{2}{|c|}{ 30-Day Status } & \multirow[t]{2}{*}{ CHR (95\% Cl) } & \multirow[t]{2}{*}{ p-value } & \multirow[t]{2}{*}{ AHR $(95 \% \mathrm{Cl})$} & \multirow[t]{2}{*}{ p-value } \\
\hline & Dead (46) & Alive (130) & & & & \\
\hline Age (in years) ${ }^{\dagger}$ & $61.3 \pm 11.1$ & $54.1 \pm 11.6$ & $1.04(1.02-1.07)$ & $0.002 *$ & $1.03(0.99-1.05)$ & 0.056 \\
\hline Rural residence (ref. urban) & $17(37.0)$ & $24(18.5)$ & $2.19(1.21-3.99)$ & $0.010^{*}$ & $1.05(0.53-2.09)$ & 0.888 \\
\hline Pre-hospital delay $>12 \mathrm{~h}$ & $4 I(89.1)$ & $78(60 \%)$ & $4.64(1.83-11.74)$ & $0.001 *$ & $4.32(1.68-11.10)$ & $0.002^{*}$ \\
\hline Atypical presentations & $28(60.9)$ & $40(30.8)$ & $2.89(1.59-5.23)$ & $<0.00 I^{*}$ & $1.49(0.79-2.79)$ & 0.215 \\
\hline Systolic BP $(\mathrm{mmHg})^{\dagger}$ & $116.5 \pm 27.8$ & $128.0 \pm 23.2$ & $0.98(0.97-0.99)$ & $0.005^{*}$ & $0.98(0.96-0.99)$ & $0.00 I^{*}$ \\
\hline Heart rate $(\mathrm{bpm})^{\dagger}$ & $100.2 \pm 35$ & $89.5 \pm 18.5$ & $1.013(1.003-1.02)$ & $0.010^{*}$ & $1.003(0.99-1.01)$ & 0.535 \\
\hline Serum creatinine $(\mathrm{mg} / \mathrm{dL})^{\dagger}$ & $1.3 \pm 0.9$ & $1.0 \pm 0.7$ & 1.18 (0.93-I.49) & 0.244 & $1.08(0.78-1.48)$ & 0.649 \\
\hline Hemoglobin $(\mathrm{mg} / \mathrm{dL})^{\dagger}$ & $12.4 \pm 2.8$ & $14.2 \pm 2.4$ & $0.84(0.77-0.92)$ & $<0.00 I^{*}$ & $0.96(0.87-1.07)$ & 0.494 \\
\hline Killip class $\geq 2$ (ref. class I) & 44 (95.7) & $62(47.7)$ & I8.I (4.38-74.6) & $<0.00 I^{*}$ & $10.69(2.54-44.95)$ & $0.00 I^{*}$ \\
\hline STEMI (ref. NSTE-ACS) & $32(69.6)$ & $75(57.7)$ & $1.61(0.86-3.02)$ & 0.137 & $1.65(0.83-3.29)$ & 0.152 \\
\hline Presence of MS & $24(52.2)$ & $38(29.2)$ & $2.260(1.26-4.02)$ & $0.006 *$ & $3.25(1.72-6.15)$ & $<0.001 *$ \\
\hline $\mathrm{EF}<40 \%$ (ref. $\geq 40 \%$ ) & $28(60.9)$ & $18(13.8)$ & $2.83(1.56-5.11)$ & $0.001 *$ & 2.59 (I.39-4.84) & $0.003 *$ \\
\hline
\end{tabular}

Notes: *p-value $<0.05$; ${ }^{\dagger}$ Expressed as mean \pm standard deviation (SD).

Abbreviations: BP, blood pressure; Bpm, beat per minute; Cl, confidence interval; EF, ejection fraction; HR, hazard ratio; NSTE-ACS, Non-ST-elevation Acute coronary syndrome; MS, metabolic syndrome; STEMI, ST-elevation myocardial infarction. 


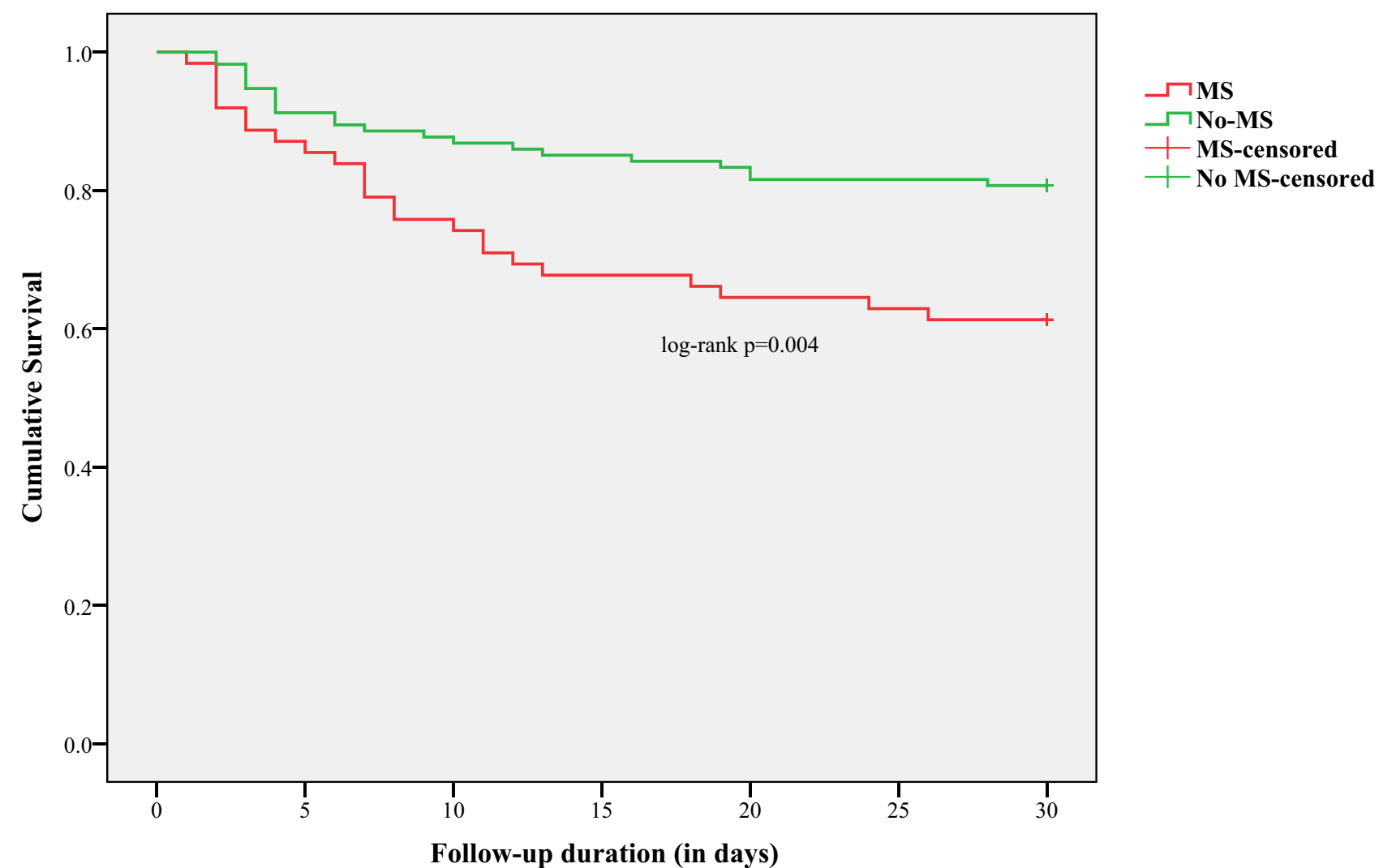

Figure 2 Kaplan Meier survival analysis of ACS patients with MetS and without MetS.

The prevalence of MS (35.2\%) among the patients with ACS in the present study is significantly higher than 11$24 \%$ observed in general populations of sub-Saharan Africa. ${ }^{5}$ However, the prevalence of MS recorded in our study participants was low compared to previous studies done among patients with ACS from six Middle East countries that reported $46 \%$ of hospitalized ACS patients had MS. ${ }^{17}$ Likewise, a study conducted among ACS patients who received coronary intervention in China showed $46 \%$ of ACS patients had MS. ${ }^{18}$ Another study done by Cavalari et $\mathrm{al}^{19}$ indicated that more than half $(62 \%)$ of ACS patients had MS. This difference might be due to differences in population-level risk factors, difference in MS diagnostic criteria used, and relatively low prevalence of MS in sub-Saharan Africa compared to other regions such as the Middle East and China. ${ }^{20,21}$

One of the major findings of the present study is that MS is significantly associated with in-hospital MACE. This finding is in line with the Analysis of the Gulf Registry of Acute Coronary Events (Gulf RACE), which demonstrated a significant association between MS and non-fatal MACE (heart failure and recurrent myocardial infarction). ${ }^{17}$ In addition, the report of a prior study done by Zeller et $\mathrm{al}^{22}$ showed a significant association between MS and severe inhospital heart failure among patients hospitalized with acute myocardial infarction. Furthermore, the association between MS and MACE sustained even on long-term followup. ${ }^{18,19,23}$ This claim could be elucidated in view of the fact that inflammation caused by this syndrome plays a critical role in the process of atherosclerosis, which causes more severe coronary artery disease, restenosis and severe angiographic stenosis based on objective finding such as Syntax and modified Gensini. ${ }^{24,25}$

The other finding and most probably the main finding of this study is that MS is an independent predictor of 30-day mortality. A significant association between MS and a range of adverse cardiovascular events including mortality were noted in previous studies. Study conducted by Al-Rasadi et $\mathrm{al}^{26}$ which enrolled $1392 \mathrm{ACS}$ patients, reported increased odds of in-hospital death among MS patients with ACS (OR, 4.42; 95\% CI: $1.25-15.5 ; \mathrm{P}=0.020$ ). Similarly, analysis of the Myocardial Ischemia Reduction with Aggressive Cholesterol Lowering (MIRACL) ${ }^{27}$ trial showed that, ACS patients with MS had around 1.5-fold increased risk of 
adverse cardiovascular events (non-fatal myocardial infarction, recurrent $\mathrm{MI}$ and cardiac arrest) including mortality. Moreover, a meta-analysis of 87 cohort studies confirmed that MS was associated with a two-fold increase in cardiovascular mortality and 1.5 fold increase in overall mortality. ${ }^{28}$ On the other hand, further studies with different duration of follow-up did not demonstrate a significant association between MS and cardiovascular or all-cause mortality particularly in ACS patients managed with PCI. ${ }^{29,30}$

The high risk of 30-day mortality $(\mathrm{HR}=3.25,95 \%$ $\mathrm{CI}=1.72-6.15)$ observed among ACS patients with MS in the present study could be explained by higher prevalence of prior history of coronary artery disease and chronic kidney disease among patients with this syndrome which might influence mortality. ${ }^{31}$ Additionally, prolonged prehospital delay and sub-optimal treatment (lack of reperfusion therapy in our setup) and small sample size might also contribute to the high mortality rate observed among ACS with MS in the present study.

The present study has several limitations. First, it included a relatively small number of ACS patients from two tertiary hospitals in Ethiopia. Second, some important predictors (, Syntax score, uric acid, and white blood cell) were missed since the data were collected from an observational study (routine clinical practice). Third, the prevalence of MS might be underestimated since the use of statin for primary prevention of cardiovascular disease is not considered as MS criteria and patients who died before biochemical measurement and confirmation of ACS diagnosis were excluded. Fourth, the findings of this study are based on data collected from two tertiary hospitals in Ethiopia and cannot be generalized for other sub-Saharan African countries. Therefore, the results of the present study should be used as a hypothesis-generating and largescale registry data needed to confirm the results.

\section{Conclusions}

The prevalence of MS among ACS patients in Ethiopia is high. MS is associated with in-hospital MACE particularly, heart failure and cardiogenic shock with a significant increase in 30-day mortality. Timely diagnosis, prevention, and management of different components of MS could improve the outcome of patients with ACS.

\section{Abbreviations}

ACEI, Angiotensin Converting Enzyme Inhibitors; ACS, Acute Coronary Syndromes; ARB, Angiotensin Receptor Blockers; DAPT, Dual Antiplatelet Therapy; FPG, Fasting plasma glucose; HDL-C, High density lipoprotein cholesterol; LDL, Low density lipoprotein cholesterol; MACE, Major Adverse cardiovascular Events; MS, Metabolic syndrome; NSTE-ACS, No-ST-elevations Acute Coronary Syndromes; STEMI, ST-elevations Myocardial infarction; PCI, Percutaneous coronary intervention.

\section{Data Sharing Statement}

The data used for this study will be made available by the corresponding author upon reasonable request.

\section{Ethics Approval and Consent to Participate}

The study protocol was approved by the Institutional Review Board (IRB) of Jimma University, Institute of Health before initiating data collection with a reference number of IHRPGD/193/18. In addition, permission was obtained from responsible bodies of both hospitals before extracting data from active patient's case records. All the study protocols were conducted in line with the ethical principle of the declaration of Helsinki. ${ }^{32}$ Written and verbal informed consent was obtained from all study participants. The confidentiality and privacy of study participant's data were maintained by using different codes throughout the data collection tools.

\section{Acknowledgments}

We would like to thank Jimma University Institute of health for financially supporting this study.

\section{Author Contributions}

All authors contributed to data analysis, drafting or revising the article, have agreed on the journal to which the article will be submitted, gave final approval of the version to be published, and agree to be accountable for all aspects of the work.

\section{Funding}

This work was supported by the Institute of Health, Jimma University [JUIH/193/10, 2018]. The funding body had no involvement in study design, data collection, analysis, interpretation, manuscript writing, and in the decision to submit the article for publication.

\section{Disclosure}

The authors declare that there is no conflict of interest regarding this work. 


\section{References}

1. Kelli HM, Kassas I. Cardio metabolic syndrome: a global epidemic. J Diabetes Metab. 2016;6(3). doi:10.4172/2155-6156.1000513

2. Ritchie SA, Connell JMC. The link between abdominal obesity, metabolic syndrome and cardiovascular disease. Nutr Metab Cardiovasc Dis. 2007;17(4):319-326. doi:10.1016/j.numecd.2006.07.005

3. Grundy SM, Cleeman JI, Daniels SR, et al. Diagnosis and management of the metabolic syndrome: an American Heart Association/National Heart, Lung, and Blood Institute scientific statement. Circulation. 2005;112 (17):2735-2752. doi:10.1161/CIRCULATIONAHA.105.169404

4. Saklayen MG. The global epidemic of the metabolic syndrome. Curr Hypertens Rep. 2018;20(2):12. doi:10.1007/s11906-018-0812-z

5. Jaspers Faijer-Westerink H, Kengne AP, Meeks KAC, Agyemang C. Prevalence of metabolic syndrome in sub-Saharan Africa: a systematic review and meta-analysis. Nutr Metab Cardiovasc Dis. 2020;30(4):547-565. doi:10.1016/j.numecd.2019.12.012

6. Gouda HN, Charlson F, Sorsdahl K, et al. Burden of noncommunicable diseases in sub-Saharan Africa, 1990-2017: results from the Global Burden of Disease Study 2017. Lancet Glob Heallth. 2019;7(10):e1375-87. doi:10.1016/S2214-109X(19)30374-2

7. Cappuccio FP, Miller MA. Cardiovascular disease and hypertension in sub-Saharan Africa: burden, risk and interventions. Intern Emerg Med. 2016;11(3):299-305. doi:10.1007/s11739-016-1423-9

8. Yuyun MF, Sliwa K, Kengne AP, Mocumbi AO, Bukhman G. Cardiovascular diseases in sub-saharan Africa compared to high-income countries: an epidemiological perspective. Glob Heart. 2020;15(1):15. doi:10.5334/gh.403

9. Ambachew S, Endalamaw A, Worede A, Tegegne Y, Melku M, Biadgo B. The prevalence of metabolic syndrome in ethiopian population: a systematic review and meta-analysis. J Obes. 2020; 2020:2020. doi:10.1155/2020/2701309

10. Ford ES. Risks for all-cause mortality, cardiovascular disease, and diabetes associated with the metabolic syndrome: a summary of the evidence. Diabetes Care. 2005;28(7):1769-1778. doi:10.2337/ diacare.28.7.1769

11. Galassi A, Reynolds K, He J. Metabolic syndrome and risk of cardiovascular disease: a meta-analysis. Am J Med. 2006;119 (10):812-819. doi:10.1016/j.amjmed.2006.02.031

12. Gami AS, Witt BJ, Howard DE, et al. Metabolic syndrome and risk of incident cardiovascular events and death. A systematic review and meta-analysis of longitudinal studies. J Am Coll Cardiol. 2007;49 (4):403-414. doi:10.1016/j.jacc.2006.09.032

13. Gurka MJ, Filipp SL, Deboer MD. Geographical variation in the prevalence of obesity, metabolic syndrome, and diabetes among US adults article. Nutr Diabetes. 2018;8(1). doi:10.1038/s41387-0180024-2

14. Thygesen K, Alpert JS, Jaffe AS, Simoons ML, Chaitman BR, White HD. Third universal definition of myocardial infarction. $J$ Am Coll Cardiol. 2012;60(16):1581-1598. doi:10.1016/j.jacc.20 12.08.001

15. Alberti KGMM, Eckel RH, Grundy SM, et al. Harmonizing the metabolic syndrome: a joint interim statement of the international diabetes federation task force on epidemiology and prevention; National heart, lung, and blood institute; American heart association; World heart federation; International. Circulation. 2009;120 (16):1640-1645. doi:10.1161/CIRCULATIONAHA.109.192644

16. Alberti KGMM, Zimmet P, Shaw J. Metabolic syndrome - A new world-wide definition. A consensus statement from the International Diabetes Federation. Diabet Med. 2006;23(5):469-480. doi:10.1111/ j.1464-5491.2006.01858.x

17. Al Suwaidi J, Zubaid M, El-Menyar AA, et al. Prevalence of the metabolic syndrome in patients with acute coronary syndrome in six middle eastern countries. J Clin Hypertens. 2010;12(11):890-899. doi:10.1111/j.1751-7176.2010.00371.x
18. Wang HH, Jia SD, Liu Y, et al. [The impact of metabolic syndrome and its individual components on long-term prognosis of patients undergoing percutaneous coronary intervention]. Zhonghua Yi Xue Za Zhi. 2020;100(21):1623-1628. doi:10.3760/cma.j.cn112137-201 90920-02077. [Chinese]

19. Cavallari I, Cannon CP, Braunwald E, et al. Metabolic syndrome and the risk of adverse cardiovascular events after an acute coronary syndrome. Eur J Prev Cardiol. 2018;25(8):830-838. doi:10.1177/ 2047487318763897

20. Ansarimoghaddam A, Adineh HA, Zareban I, Iranpour S, HosseinZadeh A, Kh F. Prevalence of metabolic syndrome in Middle-East countries: meta-analysis of cross-sectional studies. Diabetes Metab Syndr Clin Res Rev. 2018;12(2):195-201. doi:10.1016/j.dsx.2017.11.004

21. Li R, Li W, Lun Z, et al. Prevalence of metabolic syndrome in mainland China: a meta-analysis of published studies. BMC Public Health. 2016;16(1):1-10. doi:10.1186/s12889-016-2870-y

22. Zeller M, Steg PG, Ravisy J, et al. Prevalence and impact of metabolic syndrome on hospital outcomes in acute myocardial infarction. Arch Intern Med. 2005;165(10):1192-1198. doi:10.1001/archinte. 165.10.1192

23. Lovic MB, Djordjevic DB, Tasic IS, Nedeljkovic IP. Impact of metabolic syndrome on clinical severity and long-term prognosis in patients with myocardial infarction with ST-segment elevation. Hell J Cardiol. 2018;59(4):226-231. doi:10.1016/j.hjc.2018.02.002

24. Widecka K, Safranow K, Lewandowski M, Przybycień K, Gorący J, Kornacewicz-Jach Z. Angiographic severity of coronary artery disease and cardiovascular risk in acute coronary syndrome in patients with metabolic syndrome. Kardiol Pol. 2018;76(3):662-668. doi:10. 5603/KP.a2017.0259

25. Miri R, Sajjadieh A, Parsamahjoob M, et al. Relationship between metabolic syndrome and angiographic severity of coronary artery disease. ARYA Atheroscler. 2016;12(5):220-225.

26. Al-Rasadi K, Sulaiman K, Panduranga P, Al-Zakwani I. Prevalence, characteristics, and in-hospital outcomes of metabolic syndrome among acute coronary syndrome patients from Oman. Angiology. 2011;62(5):381-389. doi:10.1177/0003319710382419

27. Schwartz GG, Olsson AG, Szarek M, Sasiela WJ. Relation of characteristics of metabolic syndrome to short-term prognosis and effects of intensive statin therapy after acute coronary syndrome: an analysis of the Myocardial Ischemia Reduction with Aggressive Cholesterol Lowering (MIRACL) trial. Diabetes Care. 2005;28(10):2508-2513. doi: $10.2337 /$ diacare.28.10.2508

28. Mottillo S, Filion KB, Genest J, et al. The metabolic syndrome and cardiovascular risk: a systematic review and meta-analysis. $J$ Am Coll Cardiol. 2010;56(14):1113-1132. doi:10.1016/j.jacc.2010.05.034

29. Won KB, Kim BK, Chang HJ, et al. Metabolic syndrome does not impact long-term survival in patients with acute myocardial infarction after successful percutaneous coronary intervention with drug-eluting stents. Catheter Cardiovasc Interv. 2014;83(5):713-720. doi:10.1002/ccd.25150

30. Lee Y, Lim YH, Shin JH, Park J, Shin J, Kim KS. The impact of metabolic syndrome on clinical outcomes after everolimus-eluting stent implantation. Am J Cardiol. 2015;116(5):717-724. doi:10.10 16/j.amjcard.2015.05.041

31. Liu L, Miura K, Fujiyoshi A, et al. Impact of metabolic syndrome on the risk of cardiovascular disease mortality in the United States and in Japan. Am J Cardiol. 2014;113(1):84-89. doi:10.1016/j.amjcard. 2013.08.042

32. World Medical Association. World Medical Association Declaration of Helsinki: ethical principles for medical research involving human subjects. JAMA. 2013;310(20):2191-2194. doi:10.1001/jama.2013. 281053. 


\section{Publish your work in this journal}

Diabetes, Metabolic Syndrome and Obesity: Targets and Therapy is an international, peer-reviewed open-access journal committed to the rapid publication of the latest laboratory and clinical findings in the fields of diabetes, metabolic syndrome and obesity research. Original research, review, case reports, hypothesis formation, expert opinion and commentaries are all considered for publication. The manuscript management system is completely online and includes a very quick and fair peer-review system, which is all easy to use. Visit http://www.dovepress.com/testimonials.php to read real quotes from published authors.

Submit your manuscript here: https:/www.dovepress.com/diabetes-metabolic-syndrome-and-obesity-targets-and-therapy-journal 